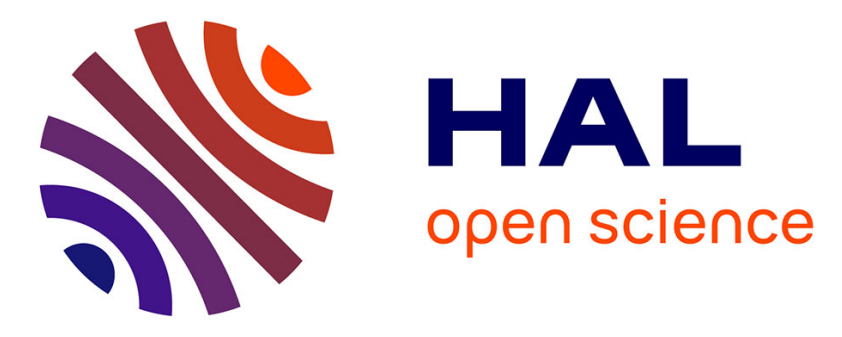

\title{
Dynamique Émotionnelle et activité: le cas des enseignants débutants
}

Luc Ria, Sébastien Chalies

\section{To cite this version:}

Luc Ria, Sébastien Chalies. Dynamique Émotionnelle et activité: le cas des enseignants débutants.

Recherche et formation, 2003, 42, pp.7 - 19. 10.3406/refor.2003.1823 . hal-01498527

\section{HAL Id: hal-01498527 \\ https://hal-univ-tlse2.archives-ouvertes.fr/hal-01498527}

Submitted on 30 Mar 2017

HAL is a multi-disciplinary open access archive for the deposit and dissemination of scientific research documents, whether they are published or not. The documents may come from teaching and research institutions in France or abroad, or from public or private research centers.
L'archive ouverte pluridisciplinaire HAL, est destinée au dépôt et à la diffusion de documents scientifiques de niveau recherche, publiés ou non, émanant des établissements d'enseignement et de recherche français ou étrangers, des laboratoires publics ou privés. 


\section{Dynamique émotionnelle et activité : le cas des enseignants débutants}

\section{Luc Ria, Sébastien Chaliès}

LIRDEF, Institut Universitaire de Formation des Maîtres de Montpellier

Article sous presse pour la Revue Recherche et Formation

\section{Résumé}

Cet article présente un cadre d'analyse des émotions situées et des extraits de l'étude des émotions d'enseignants débutants en classe ou lors de leur entretien de conseil pédagogique. A partir des trois catégories d'expérience selon Peirce (1931-1935), nous avons catégorisé les émotions des enseignants en documentant des états affectifs, des sentiments et des émotions-types. Les résultats principaux montrent et discutent a) le rôle des émotions lors de la construction de connaissances professionnelles, b) l'émergence d'émotions contradictoires dans l'activité en classe, c) la dynamique émotionnelle entre un tuteur et un enseignant débutant lors d'un entretien de conseil pédagogique. Ces résultats nous invitent à discuter l'intérêt des phénomènes émotionnels pour décrire l'activité professionnelle, notamment l'intérêt de décrire les émotions selon des modes potentiel, actuel et virtuel de l'expérience et la difficulté à évoquer ses émotions a posteriori.

Mots-clés : Emotions - Significations - Cours d'expérience - Enseignants débutants

Dans “ La modification ”, Michel Butor (1957) vous invite à prendre le train Paris - Rome en troisième classe. Votre intention est de rejoindre une maîtresse que vous retrouvez à chacun de vos voyages d'affaires pour 
vivre ensemble définitivement. Vous avez décidé de vous séparer de votre femme et de vos enfants et d'apporter ainsi une grande modification à votre vie. Vous attendez ce voyage comme une libération, comme l'espoir exaltant d'une vie nouvelle. Et pourtant pèse sur vous une lassitude, vous diriez presque un malaise. Au fil des heures de ce voyage ferroviaire et des détails de l'horizon qui défilent devant vous en continu de Paris à Rome, s'entrelacent dans votre esprit des souvenirs, des perceptions futiles, des sentiments présents et passés. Peu à peu s'estompent votre optimisme et votre emportement venant vider de son sens votre projet initial. Inexorablement s'effectue un changement d'éclairages, de perspectives et de significations, lié à la fatigue, aux circonstances de votre voyage et aux incessantes réminiscences de votre passé. A la fin du parcours, votre état d'esprit s'est tellement modifié que vous renoncez au changement même en vue duquel vous étiez parti.

Michel Butor décrit de façon subtile et détaillée la modification à la fois imperceptible et profonde du flux d'expérience d'un acteur circonscrit spatialement et temporellement au compartiment d'un train entre Paris et Rome. Pourtant de cet espace clos, son expérience se déploie sur la base de plusieurs temporalités : ses expériences passées avec sa femme et sa maîtresse et les attentes à l'égard de ses futures expériences. Ses émotions, à la fois synthèse et nœud de tension entre son passé, son présent et son futur, jouent un rôle décisif dans sa lente et inexorable modification. Nous l'interprétons à la lumière de deux dynamiques émotionnelles : la première constituée de ses expériences d'adultère sur la base desquelles émergent des sentiments d'optimisme et de confiance, la seconde relative à ses expériences maritales sur la base desquelles émergent des sentiments de doutes, de remords et de pessimisme. La modification de ses sentiments lors de son voyage résulte de cette double dynamique contradictoire : la seconde prenant le pas progressivement et implicitement sur la première.

Notre recherche s'intéresse à l'analyse du flux de l'expérience des enseignants débutants et notamment du flux de leurs émotions. Si par nature leur dynamique émotionnelle s'écarte totalement de celles décrites par Butor dans ce nouveau roman, des similitudes existent cependant concernant leur genèse, leur déploiement et leur modification au fil des événements de la classe : la classe constitue également une unité de temps et de lieu dans laquelle l'expérience présente synthétise différents éléments d'expériences passées. Notre projet est de montrer que le flux des émotions (ou dynamique émotionnelle) de ces enseignants débutants contribue fondamentalement à la modification de leur activité professionnelle.

L'enseignement apparaît pour une littérature abondante comme un travail émotionnel occasionnant des sentiments de satisfaction mais aussi de tension voire de souffrance (Par exemple : Blanchard-Laville, 2001 ; Day \& Leitel, 2001 ; Tardif \& Lessard, 1999). L'importance des émotions en classe a été particulièrement mise en évidence chez les enseignants débutants (Feiman-Nemser \& Remillard, 1996 ; Frijda, 1986 ; Huberman, 1989 ; Veenman, 1984) en soulignant notamment une période critique, relative aux cinq ou sept premières années du métier, s'accompagnant d'émotions fortes et de dilemmes (Ria \& Durand, 2001 ; Ria, Saury, Sève \& Durand, 2001). Ces recherches ont décrit les émotions des enseignants à partir de questionnaires, d'entretiens et de journaux de bord. Ces méthodes ont en quelque sorte détaché les émotions des actions professionnelles et des contextes dans lesquels elles apparaissaient.

Cet article poursuit trois objectifs : a) présenter une analyse des émotions situées selon une théorie inspirée de la sémiologie de Peirce (1931-1935), b) présenter des extraits de cours d'expérience d'enseignants débutants en classe et lors d'un entretien de conseil pédagogique, c) discuter l'intérêt de s'intéresser aux phénomènes émotionnels pour décrire l'activité professionnelle.

\section{La théorie sémiologique du cours d'expérience}

Notre étude des émotions s'inscrit dans l'approche de la cognition située, mettant en exergue la 
situativité des processus psychologiques. Les émotions sont considérées comme indissociables des situations dans lesquelles elles prennent forme.

S'appuyant sur la sémiotique de Peirce (1931-1935), la théorie sémiologique du cours d'expérience (Theureau, 1992) s'attache au niveau de l'expérience qui est significatif pour l'acteur, c'est-à-dire montrable, racontable et commentable par lui a posteriori. Cette théorie est fondée sur le postulat que le niveau de l'expérience qui est significatif du point de vue de l'acteur est un niveau d'organisation relativement autonome par rapport à d'autres niveaux possibles d'analyse de l'activité et qu'il peut donner lieu à des observations, descriptions et explications valides et utiles (Theureau, 1992).

Les émotions, selon la théorie sémiologique du cours d'expérience, sont étudiées in situ à partir de ce dont l'acteur peut rendre compte et des manifestations qu'un observateur peut percevoir. Leur description repose d'une part sur le dévoilement par l'acteur de l'environnement significatif, en permanente évolution, dans lequel il est plongé, et d'autre part, sur la description des contraintes objectives de son activité (Theureau, 1992).

\section{Les trois catégories d'expérience selon Peirce}

Peirce a distingué trois catégories d'expérience : la priméité, la secondéité et la tiercéité. Ces catégories sont à la fois inextricablement mêlées et distinctes : l'acteur éprouve son expérience de façon globale mais peut exprimer a posteriori des contenus d'expérience différents, respectivement de l'ordre de la priméité, de la secondéité et de la tiercéité. Ces trois catégories d'expérience se rattachent à trois modes d'existence au monde : le Potentiel, l'Actuel et le Virtuel (Fontanille, 1998 ; Theureau, 1992).

La priméité est la catégorie d'expérience d'un acteur tel qu'il est positivement sans référence à quoi que ce soit d'autre. Elle se caractérise par la révélation immédiate de soi au monde. Par exemple, la sensation du “ mouillé " saisie dans sa seule présence. Cette sensation syncrétique pour l'acteur ou qualia est sans actualité particulière avec son environnement significatif ou "son monde" (Varela, 1989). Par son indétermination, la priméité se rattache au mode potentiel dans la mesure où elle constitue pour l'acteur une ouverture - liée à ses expériences passées - de possibles non actualisés dans l'expérience présente.

La secondéité est la catégorie d'expérience d'un acteur lors de la concrétisation d'un fait. Elle traduit l'expérience pour l'acteur d'une interaction particulière avec son monde. Par exemple, la sensation du “ mouillé" mise en relation avec la "pluie qui tombe". La secondéité se rattache au mode actuel dans la mesure où elle est l'actualisation concrète d'un ou plusieurs possibles pour l'acteur. Par exemple, l'action de se protéger de la pluie qui tombe ou de penser que la pluie est utile pour la végétation.

La tiercéité est la catégorie d'expérience d'un acteur donnant lieu à l'élaboration de raisonnements, à la généralisation. Elle est le mode de la construction de connaissances. Par exemple, l'expérience-type d'être de mauvaise humeur quand il pleut, confirmant à l'acteur la régularité de son expérience dans des situations vécues par lui comme similaires. La tiercéité permet la typicalisation de son rapport au monde à partir des expériences passées et présentes. Elle se rattache au mode virtuel dans la mesure où elle produit et/ou modifie les connaissances de l'acteur selon un mode dégagé des contingences. 


\section{Trois niveaux de description des émotions}

À partir des trois catégories d'expérience selon Peirce, trois niveaux de description des émotions sont proposés.

Premièrement, des états affectifs syncrétiques relatifs à la priméité de l'expérience. Ils constituent le flux émotionnel continu lié à l'adaptation de l'acteur à son environnement physique et social. Ils sont la révélation immédiate et diffuse pour l'acteur de son engagement au monde. Par exemple, la sensation diffuse de bien-être ou de mal être, de confort ou d'inconfort, d'agrément ou de désagrément. Ces états affectifs enracinés dans le corps, peuvent se développer sans actualité présente ni analyse ni raisonnement.

Deuxièmement, des sentiments relatifs à la secondéité de l'expérience. Ils sont les moments saillants et limités dans le temps de ces émotions pouvant être racontés et commentés. Par exemple, l'expression du sentiment ponctuel d'agacement lié à la pluie qui tombe.

Troisièmement, des émotions-types relatives à la tiercéité de l'expérience. Elles correspondent à la typification des émotions de l'acteur. Elles participent à la construction pour l'acteur de régularités éprouvées dans des situations similaires à ses yeux. Par exemple, la connaissance (plus ou moins implicite) de sa mauvaise humeur typiquement liée aux journées pluvieuses.

Les états affectifs, les sentiments et les émotions-types composent les émotions et leurs développements forment la dynamique émotionnelle du cours d'expérience de l'acteur. Ainsi définies les émotions émergent de la manière dont l'homme appréhende le monde et interagit avec lui. Elles constituent, au même titre que les perceptions, les préoccupations, les actions et les interprétations, une des composantes de l'expérience humaine (Theureau, 1992). L'homme peut en rendre compte en dévoilant a posteriori des contenus significatifs plus ou moins élaborés, plus ou moins symbolisés. Les états affectifs se développent de façon permanente et implicite. Ils sont des états syncrétiques participant à l'ouverture des possibles pour l'acteur (mode potentiel). De l'interaction avec son monde émergent des sentiments de l'ordre de l'action, de l'impulsion (mode actuel). Et les émotions-types participent à la typification de son expérience (mode virtuel).

Les émotions revêtent divers degrés de communicabilité. Contrairement aux sentiments qui sont actuels pour l'acteur et plus facilement exprimables moyennant des conditions favorables, les états affectifs indéterminés ne sont pas directement racontables ou commentables.

Notre méthode procède d'un effort pour distinguer différents contenus émotionnels tout en postulant que l'acteur éprouve des émotions de façon unitaire et synthétique, comme engagement global au monde (Sartre, 1938). Elle consiste à favoriser l'explicitation du flux d'expérience (Dewey, 1938) de l'acteur à un grain de description le plus fin possible en fonction de ce qui fait signe pour lui, et plus particulièrement à décrire pour notre recherche les états affectifs, les sentiments et les émotions-types des enseignants débutants.

\section{Méthode}

\section{Recueil et traitement des données}

Deux catégories de données ont été recueillies : a) des données d'observation et d'enregistrement pendant l'action : l'enregistrement vidéo de l'action des enseignants et des élèves, à l'aide d'une caméra VHS sur pied et d'un micro HF, b) des données d'autoconfrontation a posteriori : l'auto-estimation des états affectifs des enseignants sur une échelle analogique en sept points à partir du visionnement de l'enregistrement vidéo, et l'enregistrement de leurs verbalisations lors d'un entretien d'autoconfrontation.

\section{La documentation des émotions}

Les états affectifs ont été documentés à partir d'une échelle d'Estimation des Etats Affectifs (ou échelle 
EEA) qui a fait préalablement l'objet d'une validation concernant la corrélation entre l'estimation de ses états affectifs et les relevés de la fréquence cardiaque comme indicateur biologique des émotions (Ria, 2001). Cette échelle permet à l'acteur d'estimer et d'exprimer synthétiquement le caractère positif ou négatif de son expérience. La fluctuation de son estimation traduit la dimension fondamentale d'adaptation de l'activité humaine recherchant des états positifs, agréables, confortables et anticipant ou évitant les situations négatives, désagréables ou inconfortables.

Cette échelle EEA est constituée de sept points : de +3 (très agréable ou très confortable) à -3 (très désagréable ou très inconfortable). L'estimation sur cette échelle de la tonalité de ses états affectifs correspond à un ressenti syncrétique et immédiat. Il s'agit du niveau seulement montrable des émotions. L'estimation par les enseignants du caractère positif ou négatif de leur expérience en classe s'est déroulée immédiatement après la leçon en visionnant la vidéo. Ils pouvaient à tout instant modifier les valeurs estimées sur l'échelle EEA sans avoir à les justifier.

A la suite de l'estimation synthétique des états affectifs en classe, les sentiments ont été documentés lors d'un entretien d'autoconfrontation. Cette documentation a consisté à demander aux enseignants, confrontés à l'enregistrement vidéo de leur activité en classe, de décrire et commenter pas à pas leur expérience telle qu'ils l'ont vécue et ressentie. Ils étaient incités à décrire ce qu'ils faisaient, ce qu'ils pensaient, ce qu'ils prenaient en compte pour agir, ce qu'ils percevaient ou ressentaient. Il s'agit du niveau racontable et commentable des émotions qui émerge à partir de ce qui fait signe pour l'acteur dans la situation. L'étiquetage des sentiments s'est faite à partir des données d'autoconfrontation sans reprendre systématiquement la nomination des enseignants (e.g. : L'expression “ Là, je ne me sens pas terrible !” lors de l'autoconfrontation étiquetée comme le sentiment d'un malaise).

Les émotions-types ont également été documentées à partir de l'entretien d'autoconfrontation, grâce à la mise en évidence de la récurrence de certaines émotions éprouvées par les enseignants. Il s'agit du niveau de typicalisation des émotions. L'étiquetage des émotions-types s'est faite à partir de l'explicitation par les enseignants d'expériences vécues dans des situations estimées par eux comme similaires. (e.g. : “ C'est toujours difficile de les mettre en action !') traduisant la régularité des émotions éprouvées par l'enseignant dans des situations identiques.

L'intégralité des verbalisations des enseignants et des élèves en classe a été retranscrite verbatim, et leurs comportements ont été décrits systématiquement. Cette description, s'est faite par un recours à des catégories permettant à un lecteur d'accéder à un niveau de compréhension fonctionnelle des situations d'enseignement, c’est-à-dire en recourant à des énoncés qui ne soient ni des généralités (e.g. : “ Parle aux élèves ") ni des paraphrases.

\section{Résultats}

Les résultats présentent, à partir d'extraits de cours d'expérience d'enseignants débutants en classe et lors d'un entretien de conseil pédagogique, la façon dont nous décrivons et analysons les émotions en situation. Les enseignants stagiaires volontaires, effectuant leur année pré-professionnelle au cours de laquelle, en tant que lauréats du concours du Professorat d'Education Physique (EP), prenaient en charge des classes et bénéficiaient d'entretiens de conseil pédagogique sous la responsabilité d'un tuteur. Au moment de l'étude, ces enseignants avaient une expérience cumulée d'enseignement d'environ trois à cinq mois.

Les trois extraits suivants mettent en évidence successivement a) le rôle des émotions dans la construction de connaissances professionnelles, b) l'émergence d'émotions contradictoires en classe, c) la 
dynamique émotionnelle entre un tuteur et un enseignant débutant lors d'un entretien de conseil pédagogique.

\section{Extrait 1 : le rôle des émotions dans la construction de connaissances professionnelles}

L'Extrait 1 débute lorsque Maud met en place sa deuxième leçon sur un cycle de huit en Tennis de table avec une classe de trente filles de $4^{\mathrm{e}}$ réparties sur huit tables. Son projet est de passer à chaque table, de lire la fiche de résultats des élèves pour choisir un exercice adapté au jeu de chacun. Il repose sur la conviction, maintes fois répétée en formation initiale, qu'un dispositif pédagogique articulant plusieurs exercices choisis en fonction du niveau et du rythme de travail des élèves permet à tous un apprentissage différencié.

Maud s'aperçoit rapidement que son projet est difficile à mettre en œuvre : quand elle arrive à une table, il lui faut interpréter la fiche de résultats, sans délai de réflexion, pour comprendre ce qu'ont fait des élèves qu'elle n'a pas eu le temps de voir jouer. Lors de l'autoconfrontation, elle dit : “ Je passe d'une table à l'autre mais je ne vois pas ce qui se passe... Alors j'essaye de me faire une idée de la façon dont elles ont dû jouer... Ce n'est pas comme si j'avais fait l'observation moi-même..." Maud indique la valeur de +1 sur l'échelle EEA. Elle éprouve un sentiment d'indécision devant la nouveauté de ce type d'intervention : " J'essaye de me montrer convaincante... Pourtant je suis assez indécise..."

$\mathrm{Au}$ fur et à mesure de ses interventions aux différentes tables, Maud se rend compte que toutes les élèves ne travaillent pas : celles des tables précédentes (à qui elle a donné les consignes d'un nouvel exercice) la sollicitent de nouveau pour des informations supplémentaires, alors que, devant elle, les autres élèves inactives s'impatientent. Maud indique alors la valeur de -1 sur l'échelle EEA. Elle éprouve des sentiments d'agacement et de lassitude à force de répéter les consignes à chaque table : “Je commence à être perturbée par le fait d'avoir à ré-expliquer... Je ne le montre pas trop, mais ça m'agace ... "Aux dernières tables, elle exprime le sentiment d'être débordée par les sollicitations qui vont en s'accroissant : "À ce moment-là, je me sens débordée en répondant aux questions des élèves..." Elle indique la valeur de -2 sur l'échelle EEA.

La connaissance relative à l'intérêt de différencier sa pédagogie et mobilisée par Maud pour structurer son intervention en classe perd peu à peu de sa pertinence au fur et à mesure qu'elle éprouve des sentiments d'indécision, de doute et d'inconfort lors de son activité de table en table. Maud ne parvient pas à enrayer l'agitation des élèves et s'interroge : “Là, je commence à me dire : Punaise ! Je n'aurais pas dû prendre ce mode de fonctionnement là..." Pourtant, Maud préfère encore endurer le caractère désagréable de son expérience plutôt que de changer inopinément de dispositif pédagogique : "Je ne veux pas me compliquer en changeant la façon de présenter ou en donnant un nouvel exercice... C'est dur de changer en fin de cours ... C'est toujours usant... Et là, j'ai déjà du mal à gérer la situation... " Maud a déjà éprouvé de façon désagréable depuis le début d'année scolaire la présentation de nouveaux exercices en fin de cours. Son intention de maintenir son dispositif pédagogique malgré l'agitation des élèves s'origine sur le fond des sentiments d'usure et d'inefficacité déjà éprouvés dans ce type de situation.

Chaque expérience apporte son lot d'éléments nouveaux modifiant les connaissances acquises au préalable et/ou permettant la construction de nouvelles connaissances. La pertinence que les enseignants leur accordent est liée à la façon dont ils les éprouvent émotionnellement et leur donnent un sens lors des situations de classe. Ainsi, les émotions constituent le flux ininterrompu et sensible de nos expériences et participent à la construction de connaissances. Cette construction ne s'effectue pas de manière froide et rationnelle indépendamment d'une expérience dans un contexte singulier et marquée par l'émergence de phénomènes émotionnels plus ou moins implicites sur le fond de résidus émotionnels éprouvés précédemment dans des 
situations typiques pour l'acteur. La coloration émotionnelle positive ou négative de l'expérience contribue alors à augmenter ou à diminuer la validité des connaissances mobilisées dans l'action. Et ce degré de validité ou d'invalidité oriente les actions futures, sans pour autant être définitif : il peut à nouveau fluctuer au cours d'autres expériences, dans des contextes différents.

\section{Extrait 2 : l'émergence d'émotions contradictoires en classe}

L'Extrait 2 débute lorsque Stéphanie demande aux élèves de s'assoire au début de la deuxième leçon d'EPS sur un cycle de six de Gymnastique avec une classe de 25 élèves de $6^{\text {ème }}$. Les élèves s'assoient bruyamment. Stéphanie les observe en restant immobile. Elle dit lors de l'autoconfrontation : "Là je regarde les deux élèves qui sont mes têtes de classe (Tristan et Mickaël) qui ont tendance à embarquer la classe... Là, ils sont déjà agités... Il faut que j'arrive dès le départ à les recadrer... Il va falloir être vigilante, sinon, si je les laisse faire, cela peut devenir bien plus dur à contrôler..."

Stéphanie indique la valeur de -1 sur l'échelle EEA. Elle exprime le sentiment d'être sur le qui-vive avec une classe difficile à contrôler notamment Tristan et Mickaël. Elle essaye dès leur prise en main d'estimer leur état d'agitation et par là-même sa propre difficulté à pouvoir les contrôler lors de cette leçon. La tonalité inconfortable de son expérience en début de leçon s'ancre pour elle sur deux éléments significatifs : l'observation présente de l'agitation de ses deux élèves leaders (lui confirmant la nécessité d'un contrôle immédiat pour garantir le bon déroulement de sa leçon), et les souvenirs désagréables de difficultés récurrentes pour les contrôler depuis le début de l'année scolaire.

Stéphanie demande à Tristan de prendre en main la démonstration des exercices d'étirement à réaliser collectivement. Elle dit lors de l'autoconfrontation: "J'ai l'habitude de le responsabiliser bien qu'il fasse souvent l'imbécile... Il peut être très efficace au sein d'un groupe ". Tristan se positionne face à la classe en grimaçant et commence à montrer les exercices d'étirement à réaliser. Stéphanie les mains sur les hanches observe la classe quelques pas en retrait vis-à-vis de Tristan : “Je vois Tristan en train de faire le singe devant la classe... J'ai envie de rire de ses pitreries... Je sais qu'il me teste en m'observant sur le côté... Donc, j'essaye de garder une position très sérieuse, très sérieuse... Je le connais... Il me teste et je dois garder mon sérieux pour ne pas rire avec lui... Sinon, je prends le risque de perdre le contrôle de la classe..."

Stéphanie éprouve lors de cet extrait des émotions contradictoires. Elle est amusée par les pitreries de Tristan et à la fois inquiète et sur la défensive. Elle indique toujours la valeur de -1 sur l'échelle EEA. Comme si l'état désagréable de son expérience lié à la difficulté de contrôler cette classe depuis le début d'année se prolongeait et contribuait à minimiser voire à annihiler le déploiement de sentiments positifs dans la situation présente (pouvant potentiellement prêter à rire).

Deux dynamiques émotionnelles, selon des échelles temporelles différentes, caractérisent l'expérience de Stéphanie. La première est relative à la typification de ses expériences, c'est-à-dire aux émotions-types, constituées d'états affectifs-types et de sentiments-types, éprouvées par elle depuis le début de l'année avec cette classe. Cette dynamique se prolonge lentement avec inertie et adhérence. Elle déborde les expériences desquelles elle a émergé et infiltre son expérience présente. La deuxième dynamique, relative à des sentiments contingents, est plus étroitement associée aux événements vécus lors de l'expérience présente.

Un déphasage apparaît entre ces deux dynamiques émotionnelles se traduisant, chez Stéphanie, par le déploiement (minimisé) de sentiments potentiellement positifs (les pitreries d'un élève) sur la base d'états affectifs marqués par une tonalité inverse (la difficulté à contrôler les élèves depuis le début d'année). Ce 
déphasage est lié à un phénomène d'hystérésis correspondant au prolongement au cœur de son expérience présente d'émotions éprouvées précédemment. Les états affectifs négatifs des expériences passées de Stéphanie viennent infiltrer de façon plus ou moins diffuse son expérience présente et lui conférer une tonalité négative et anxiogène.

L'étude des phénomènes émotionnels de Stéphanie lors de ce bref extrait nous permet de repérer la nature de sa préoccupation principale en début de leçon : le contrôle de ses élèves. Cette préoccupation constitue le fonds culturel de son activité associé à des émotions-types depuis le début de son expérience professionnelle.

\section{Extrait 3 : la dynamique émotionnelle entre un tuteur et un enseignant débutant lors d'un} entretien de conseil pédagogique

L'Extrait 3 concerne l'interaction entre un enseignant débutant (Sylvain) et son tuteur lors du 5 ème entretien de conseil pédagogique depuis le début de l'année scolaire. La $3{ }^{\text {ème }}$ leçon en Athlétisme d'un cycle de 7, achevée quelques instants au préalable, sert de support à cet entretien.

Au début de l'entretien de conseil pédagogique, Sylvain dit : “ Donc là où j'ai du mal en fait, c'est d'être capable de jongler sur l'ensemble des quatre ateliers et d'être pertinent sur chacune de mes consignes..." Son tuteur poursuit : "Oui, mais ça bon... Moi je trouve ça bien parce que par rapport à la première fois où je t'avais vu [premier entretien de conseil pédagogique], tu sais en Badminton ? [...] Là, c'est intéressant parce que tu es dans le travail avec les élèves. Tu essayes de leur donner des consignes..."

L'entretien de conseil pédagogique a été suivi par deux entretiens d'autoconfrontation conduits séparément avec Sylvain puis son tuteur. Chacun a alors évoqué ce point de désaccord survenu lors de l'entretien de conseil pédagogique.

Sylvain est peu satisfait de sa leçon et s'étonne de l'estimation faite lors de l'entretien de conseil pédagogique par son tuteur sur son intervention : “... Je suis quand même étonné parce qu'en fait dans ma tête c'était encore... ". Il exprime des sentiments plutôt négatifs et le "encore " montre le caractère récurrent de son insatisfaction. Il estime avoir eu lors de cette leçon des difficultés à superviser simultanément les différents ateliers et à donner les consignes adéquates aux élèves. De son côté, son tuteur exprime plutôt des sentiments inverses. Il estime son intervention de façon plus positive et souligne sa plus grande implication vis-à-vis de ses élèves comparativement à la dernière leçon visitée.

Le désaccord entre les deux protagonistes peut être interprété en analysant au sein du cours d'expérience collectif relatif à l'entretien de conseil pédagogique, la dynamique émotionnelle inter-individuelle qui s'y développe. Cette dynamique est constituée de sentiments ponctuels contradictoires mais aussi de phénomènes émotionnels résiduels liés à des expériences passées, partagées ou non par Sylvain et son tuteur. Elle se déploie dans l'interaction du conseil pédagogique mais aussi sur la base d'éléments résiduels mêlant synthétiquement plusieurs expériences passées, singulières ou typiques.

Pour Sylvain : a) l'expérience avec les élèves support de l'entretien présent, b) l'expérience typicalisée des entretiens précédents de conseil pédagogique depuis le début de l'année scolaire, c) l'expérience typicalisée depuis le début d'année scolaire avec les élèves de cette classe sans son tuteur. Pour son tuteur : a) l'intervention présente de Sylvain en classe, b) l'intervention de Sylvain lors de la première leçon visitée, c) les entretiens précédents de conseil pédagogique avec Sylvain, d) son expérience typicalisée des entretiens de conseil pédagogique avec des enseignants débutants en début d'année scolaire, e) sa propre expérience d'enseignement.

Nous estimons que les états affectifs plutôt négatifs éprouvés par Sylvain lors de la première leçon 
visitée, puis lors des autres entretiens de conseil pédagogique ont tendance à minimiser l'émergence de sentiments positifs lors de l'entretien présent et au contraire à renforcer chez lui l'émergence de sentiments négatifs même si, pour Sylvain, son insatisfaction est de nature différente comparativement à la première leçon visitée. Pour son tuteur, plusieurs éléments favorisent l'expression de sentiments positifs : a) les progrès depuis la première leçon visitée effectués par Sylvain au niveau de son intervention, b) l'expression du sentiment négatif de Sylvain, dès le début de l'interaction : “ Donc là où j'ai du mal en fait..." Sa façon d'intervenir positivement repose sur la conviction - acquise par l'expérience en tant que tuteur au fil des entretiens avec des stagiaires - qu'il est indispensable d'encourager un enseignant débutant surtout si celui-ci s'estime être en difficulté : “ Il se juge négativement là... [...] J'essaie de partir sur quelque chose de positif là... Dire "ça c'est bien ", c'est une bonne introduction là... " Pour ce faire, il relève les progrès effectués par ce dernier depuis le début d'année.

Cette interaction ponctuelle lors d'un entretien de conseil pédagogique nous montre la façon dont un enseignant débutant et son tuteur interprètent différemment une activité professionnelle : l'un l'ayant vécue plutôt négativement ; l'autre lui donnant une tonalité plutôt positive. Le tuteur tente de modifier la tonalité émotionnelle de son stagiaire pour instaurer une dynamique de travail favorable à la réflexion : "J'essaye un peu de le mettre en confiance pour qu'il s'engage vraiment dans la discussion et puis qu'on puisse vraiment réfléchir ensemble aux problèmes rencontrés". Il sait par expérience que les conseils pédagogiques sont vécus régulièrement par les stagiaires de façon négative : "Dans ces entretiens de conseil pédagogique, [...] On est toujours sur des choses qui posent problème..." Il estime également que sa visite est souvent prise par les stagiaires comme un jugement qui pèse sur eux. Ainsi, d'après lui, le conseil pédagogique a pour fonction ici de minimiser chez l'enseignant débutant l'émergence de sentiments négatifs en essayant d'élargir son " monde de signification ” et en l'aidant notamment à repérer des éléments positifs dans son intervention.

\section{Discussion générale}

La discussion porte sur deux points : a) l'intérêt de décrire les émotions selon les modes potentiel, actuel et virtuel, b) L'évocation des émotions lors de l'autoconfrontation.

\section{Les émotions selon les modes potentiel, actuel et virtuel}

Les émotions ne se réduisent pas à l'expression par l'acteur de simples formes conséquentes de ses actions ou décisions et contingentes à la seule situation présente. Elles sont au contraire l'essence même de l'activité humaine. Les trois extraits présentés ci-dessus montrent leur rôle au sein de l'activité d'enseignants débutants a) lors de la construction de connaissances professionnelles : les sentiments éprouvés par Maud en situation participent à l'invalidation d'une connaissance mobilisée dans l'action, b) lors d'un face à face avec des élèves difficiles : le prolongement chez Stéphanie d'états affectifs anxiogènes depuis le début d'année scolaire contribue à minimiser l'émergence de sentiments potentiellement positifs au sein de son expérience présente, c) lors d'un entretien de conseil pédagogique : les conditions favorables de collaboration reposent essentiellement sur l'instauration de la part du tuteur d'une dynamique émotionnelle positive pour minimiser le prolongement d'émotions négatives au sein de l'expérience présente du stagiaire.

Les émotions décrites précédemment émergent chez les trois enseignants de leur activité à la fois physique, mentale et sociale, c'est-à-dire des connaissances qu'ils mobilisent et de la façon dont ils interprètent les agissements de leurs élèves en classe ou encore l'interaction avec leur tuteur. Les émotions ne sont pas seulement des phénomènes internes et individuels, mais des propriétés fondamentalement relationnelles qu'il est nécessaire d'étudier sans les détacher des préoccupations et des connaissances que chacun mobilise dans 
l'action. La dynamique émotionnelle inter-individuelle peut en effet se déployer à partir de préoccupations divergentes a) dans l'Extrait 2, Stéphanie a la ferme intention de contrôler sa classe dès le début du cours et Tristan l'intention de dissiper ses camarades, b) dans l'Extrait 3, Sylvain souhaite bénéficier de l'aide pour dépasser ses difficultés d'intervention alors que tuteur a l'intention de le laisser réfléchir sur son activité en classe sans lui apporter d'aide immédiate.

L'étude des émotions selon la théorie sémiologique du cours d'expérience distingue trois modes d'existence au sein de l'expérience : les modes potentiel, actuel et virtuel. Ces modes recouvrent trois temporalités : a) le mode potentiel correspond aux émotions éprouvées précédemment lors du même cours d'expérience ou d'un autre, b) le mode actuel aux émotions éprouvées ici et maintenant, c) le mode virtuel à la typification des émotions délimitant l'orientation future de l'expérience. Selon cette acception, les résidus émotionnels (états affectifs) des expériences passées forment le mode potentiel des émotions de l'expérience présente. Ces émotions peuvent s'infiltrer de façon plus ou moins implicite selon ce qui fait signe dans la situation, et participer au mode actuel des émotions (sentiments). Ces émotions actuelles, synthétisées selon un mode virtuel détaché de toute contingence (émotions-types), deviennent les émotions potentielles et participent à la délimitation des expériences futures. Cette délimitation du futur répond à la tendance très générale chez l'homme à éviter les situations désagréables ou tout au moins à les anticiper pour minimiser leurs effets sans pour autant que ses émotions déterminent systématiquement ses décisions et comportements.

Quand bien même ces trois modes d'existence sont inextricablement mêlés au sein de l'expérience, les extraits de cours d'expérience présentés pour cet article mettent en évidence la prédominance d'un des modes d'existence en phase ou en déphasage avec les autres.

Dans l'Extrait 1, le mode actuel des émotions de Maud prédomine : l'émergence de sentiments dans la situation présente tend à diminuer peu à peu la validité de la connaissance relative à l'intérêt de différencier sa pédagogie.

Dans l'Extrait 2, le mode potentiel des émotions éprouvées précédemment par Stéphanie prédomine : les états affectifs passés et négatifs s'actualisent au sein de son expérience présente et contribuent à diminuer des sentiments de tonalité inverse.

Dans l'Extrait 3, le mode actuel (la leçon vécue quelques instants avant) des émotions de Sylvain prédomine sur la base du mode potentiel (les émotions négatives et récurrentes depuis les premières visites); chez son tuteur, le mode virtuel prédomine (les émotions-types des stagiaires lors des entretiens de conseil pédagogique). Ce qui explique en partie leur différence de point de vue : le premier " est encore dans la leçon ", affecté par l'expérience qu'il vient de vivre et qui se prolonge lors de l'entretien ; le second " est détaché des événements de la leçon ", davantage inscrit dans une réflexion dont l'empan temporel est plus large.

La distinction de trois modes d'existence des émotions d'un acteur rend plus difficile l'identification de leur genèse dans la mesure où l'étude de son cours d'expérience à l'instant t n'est pas suffisante. D'autant plus que ce cours d'expérience ne cesse de se modifier et de modifier en retour ses émotions. De ce point de vue, un paradoxe peut apparaître : si par essence les émotions sont des phénomènes privés, intimes et renouvelés en permanence - un acteur n'éprouvant jamais d'émotions identiques stricto sens - , comment rendre compte alors de façon typique de ses émotions et de celles d'une communauté d'acteurs? Le dépassement de ce paradoxe nécessite, à partir de phénomènes émotionnels intimes, de repérer des éléments de généralité communs et partageables de plusieurs cours d'expérience relatifs à un ou plusieurs acteurs. Concrètement, nous procédons à la description pour chaque enseignant des éléments typiques de sa propre dynamique émotionnelle, puis dans un 
deuxième temps, au repérage des phénomènes émotionnels récurrents des enseignants débutants à partir de la comparaison systématique de leurs états affectifs, leurs sentiments et leurs émotions-types au sein des différents cours d'expérience.

\section{L'évocation des émotions lors de l'autoconfrontation}

L'évocation des émotions, selon notre inscription théorique et notre méthode, s'effectue à partir d'un entretien d'autoconfrontation. Mais, cette évocation pose problème.

En faire part a posteriori nécessite souvent un effort pour l'acteur, voire une période d'apprentissage.

L'autoconfrontation n'est pas seulement la remémoration d'une expérience émotionnelle, comme s'il s'agissait de la seule " retraversée de l'expérience ". Elle est un nouveau déploiement émotionnel prenant ancrage sur l'expérience première. Et ce nouveau déploiement constitue une nouvelle expérience émotionnelle, adressée à autrui, qui déborde l'expérience initiale. En effet, l'autoconfrontation constitue une deuxième interaction entre l'enseignant et le chercheur relativement à la première qui était celle de l'enseignant avec ses élèves. La seconde entre l'enseignant et le chercheur se développant sur la base de la première par le biais des traces de l'activité précédente enregistrées sur vidéo.

Des états affectifs, des sentiments et des émotions-types éprouvés par l'enseignant lors de la deuxième expérience se déploient à partir de l'expérience de deux interactions : l'une avec les élèves, l'autre avec le chercheur et les élèves présents sur l'image vidéo. L'expérience émotionnelle de l'acteur est alors constituée de contenus émotionnels de la première expérience et d'éléments nouveaux de la deuxième : a) des contenus émotionnels remémorés de la première expérience avec les élèves. Par exemple, l'enseignant peut se souvenir, lors de l'autoconfrontation, du caractère plus ou moins confortable de son expérience en classe en revoyant à la vidéo des épisodes particulièrement significatifs à ses yeux, b) des contenus émotionnels émergeant lors de l'autoconfrontation, proches de ceux vécus initialement dans la situation d'enseignement. Ils émergent de ce qui fait signe (de nouveau) pour l'acteur dans la situation vécue (de nouveau) par le biais de l'enregistrement vidéo. Il s'agit ici d'un processus d'émergence d'émotions hic et nunc, proche du premier processus lors de l'expérience avec les élèves, et non d'un processus de restitution mnémonique des émotions éprouvées initialement. Par exemple, l'enseignant peut éprouver de nouveau de l'énervement en percevant (de nouveau) les comportements, les mimiques des élèves dans la situation, c) des contenus émotionnels nouveaux, produits de la deuxième expérience avec le chercheur et les élèves sur la vidéo. Par exemple, les états affectifs, les sentiments du chercheur, en fonctions de ses propres attentes et préoccupations, peuvent participer au développement d'une expérience émotionnelle avec l'enseignant débordant l'expérience émotionnelle de ce dernier avec les élèves.

Là encore, l'évocation de ses émotions lors d'une autoconfrontation mobilise implicitement une pluralité d'expériences enchevêtrées. Elle n'est pas la restitution in extenso des émotions d'une seule expérience passée, mais la synthèse à l'instant $\mathrm{t}$ - selon des processus de réminiscence, de ré-émergence et de nouvelle émergence - de différents éléments émotionnels d'expériences passées et présente. 


\section{Références}

Blanchard-Laville, Cl. (2001). Les enseignants entre plaisir et souffrance. Paris : Presse Universitaire de France.

Butor, M. (1957). La modification. Paris : Editions de Minuit.

Damasio, A.R. (1999). Le sentiment même de soi. Corps, émotions, conscience. Paris : Edition Odile Jacob.

Day, C, \& Leitel, R. (2001). Teachers' and teacher educators' lives : The role of emotion. Teaching and Teacher Education, 17 (4), 403-415.

Dewey, J. (1938/1986). Logic : The theory of inquiry. In J.A. Boydston (Ed.), John Dewey : The later works, Vol 12 (pp.1-527). Carbondale : Southern Illinois University Press. (Original work published 1938).

Feiman-Nemser, S., Rémillard, J. (1996). Perspectives on learning to teach. In F.B. Murray (Ed.), The teacher educator's handbook : Building a knowledge base for the preparation of teachers (pp.63-91). San Francisco : Jossey Bass.

Fontanille, J. (1998). Sémiotique du discours. Limoges : Presses Universitaires de Limoges.

Frijda, N.H. (1986). The Emotions. Cambridge : Cambridge University Press.

Huberman, M., (1989). La vie des enseignants : évolution et bilan d'une profession. Neuchatel : Delachaux et Niestlé.

Peirce, C.S. (1931-1935). The collected papers of C.S. Peirce. Cambridge MA : Havard University Press.

Ria, L. (2001). Les préoccupations des enseignants débutants en Education Physique et Sportive. Étude de l'expérience professionnelle et conception d'aides à la formation. Thèse de doctorat STAPS non publiée. Université de Montpellier 1.

Ria, L., \& Durand, M. (2001). Les préoccupations et la tonalité émotionnelle des enseignants débutants lors de leurs premières expériences en classe. Les Dossiers des Sciences de l'Education, 5, 111-123.

Ria, L., Saury, J., Sève, C., \& Durand, M. (2001). Les dilemmes des enseignants débutants : Etudes lors des premières expériences de classe en Education. Science et Motricité, 42, 47-58.

Sartre, J.P. (1938). Esquisse d'une théorie des émotions. Paris : Editions Herman.

Tardif, M., Lessard, C. (1999). Le travail enseignant au quotidien. Bruxelles : Editions De Boeck.

Theureau, J. (1992). Le cours d'action : Analyse sémio-logique. Essai d'une anthropologie cognitive située. Berne : Peter Lang.

Varela, F. (1989). Autonomie et connaissance. Essai sur le vivant. Paris : Seuil.

Veenman, S. (1984). Perceived Problems of Beginning Teachers. Review of Educational Research, 54(2), 143-178. 